Homology, Homotopy and Applications, vol.16(2), 2014, pp.143-158

\title{
UNIVERSAL ENVELOPING CROSSED MODULE OF A LIE CROSSED MODULE
}

\author{
JOSÉ MANUEL CASAS, RAFAEL F. CASADO, EMZAR KHMALADZE \\ AND MANUEL LADRA
}

(communicated by Graham Ellis)

\begin{abstract}
We construct a pair of adjoint functors between the categories of crossed modules of Lie and associative algebras, which extends the classical one between the categories of Lie and associative algebras. This result is used to establish an equivalence of categories of modules over a Lie crossed module and its universal enveloping crossed module.
\end{abstract}

\section{Introduction}

The general concept of a crossed module originates in the work of Whitehead in the late 1940s [25]. Namely, it was introduced as an algebraic model for a pathconnected CW-space whose homotopy groups are trivial in dimensions $>2$. There, the crossed modules were crossed modules of groups and since their introduction they have played an important role in homotopy theory. For illustration, we mention various classification problems for low-dimensional homotopy types and derivation of van Kampen theorem generalizations (see the survey of Brown [5]).

Crossed modules of Lie algebras, hereafter called Lie crossed modules, and crossed modules of associative algebras are well known, at least as analogues of crossed modules of groups in other categories.

Lie crossed modules have been investigated by various authors. Namely, in [19] Kassel and Loday use Lie crossed modules as computational tools in order to give an interpretation of the third relative Chevalley-Eilenberg cohomology of Lie algebras. Guin [17] has developed low-dimensional non-abelian cohomology of Lie algebras with coefficients in Lie crossed modules, which later has been extended to higher dimensions in [18]. Internal (cotriple) homology and Chevalley-Eilenberg homology theories of Lie crossed modules are investigated in $[\mathbf{8}, \mathbf{1 3}]$. Lie crossed modules also occurred in the "categorification" problem of the theory of Lie algebras $[\mathbf{1}]$ as an equivalent formulation of strict Lie 2-algebras (a two-dimensional generalization of the concept of Lie algebra).

Received September 11, 2013, revised December 9, 2013, May 21, 2014; published on August 1, 2014. 2010 Mathematics Subject Classification: 17B35, 18A40, 16W25, 16W99.

Key words and phrases: crossed module, Lie algebra, associative algebra, universal enveloping algebra.

Article available at http://dx.doi.org/10.4310/HHA.2014.v16.n2.a7

Copyright (C) 2014, International Press. Permission to copy for private use granted. 
The theory of crossed modules of associative algebras is not so well developed. However, in the works of Dedecker and Lue $[\mathbf{1 1}, \mathbf{2 1}]$, crossed modules of associative algebras have played a central role in what must be coefficients for low-dimensional nonabelian cohomology. Baues and Minian [2] have used them to represent the Hochschild cohomology of associative algebras. In the recent article [12], the Hochschild and (cotriple) cyclic homologies of crossed modules of associative algebras have been constructed and investigated.

The aim of the present paper is to give an interplay between crossed modules of Lie and associative algebras. Motivated by the well-known classical fact that the universal enveloping algebra functor is left adjoint to the Liezation functor, we extend this adjunction to the categories of crossed modules of Lie and associative algebras in such a way that the well-known equivalence of module structures over a Lie algebra and its universal enveloping algebra is preserved.

Our constructions can be applied to develop (co)homology theories with coefficients for crossed modules of associative and Lie algebras, and to extend for crossed modules the well-known isomorphism between homology of a Lie algebra and Hochschild homology of its universal enveloping algebra (see for example [6, Chapter XIII, Theorem 5.1]).

Notations and conventions. Throughout the paper we fix a commutative ring $K$ with unit. Lie algebras are considered over $K$ and their category is denoted by Lie. Algebras are (not necessarily unital) associative algebras over $K$ and their category is denoted by Alg.

\section{Crossed modules in Lie and associative algebras}

In this section we recall some necessary notions about crossed modules of Lie and associative algebras and describe actions for Lie crossed modules.

\subsection{Lie crossed modules and cat $^{1}$-Lie algebras}

Let $M$ and $P$ be two Lie algebras. By an action of $P$ on $M$ we mean a $K$-bilinear map $P \times M \rightarrow M,(p, m) \mapsto{ }^{p} m$ satisfying

$$
{ }^{\left[p, p^{\prime}\right]} m={ }^{p}\left({ }^{p^{\prime}} m\right)-{ }^{p^{\prime}}\left({ }^{p} m\right), \quad{ }^{p}\left[m, m^{\prime}\right]=\left[{ }^{p} m, m^{\prime}\right]+\left[m,{ }^{p} m^{\prime}\right],
$$

for all $m, m^{\prime} \in M$ and $p, p^{\prime} \in P$. In other words, the action of $P$ on $M$ is a Lie homomorphism $P \rightarrow \operatorname{Der}(M)$ to the Lie algebra of derivations of $M$. For example, if $P$ is a Lie subalgebra of some Lie algebra $Q$, and if $M$ is an ideal in $Q$, then the Lie bracket in $Q$ yields an action of $P$ on $M$.

Given a Lie action of $P$ on $M$ we can form the semi-direct product Lie algebra, $M \rtimes P$, with the underlying module $M \oplus P$ and the Lie bracket given by

$$
\left[(m, p),\left(m^{\prime}, p^{\prime}\right)\right]=\left(\left[m, m^{\prime}\right]+{ }^{p} m^{\prime}-{ }^{p^{\prime}} m,\left[p, p^{\prime}\right]\right), \quad(m, p),\left(m^{\prime}, p^{\prime}\right) \in M \oplus P .
$$

We recall from [19] that a Lie crossed module $(M, P, \mu)$ is a Lie homomorphism $\mu: M \rightarrow P$ together with an action of $P$ on $M$ such that, for all $m, m^{\prime} \in M$ and $p \in P$,

$$
\mu\left({ }^{p} m\right)=[p, \mu(m)], \quad{ }^{\mu(m)} m^{\prime}=\left[m, m^{\prime}\right] .
$$

A morphism of Lie crossed modules $(\alpha, \beta):(M, P, \mu) \rightarrow\left(M^{\prime}, P^{\prime}, \mu^{\prime}\right)$ is a pair of Lie 
homomorphisms $\alpha: M \rightarrow M^{\prime}, \beta: P \rightarrow P^{\prime}$ such that $\mu^{\prime} \alpha=\beta \mu$ and $\alpha\left({ }^{p} m\right)={ }^{\beta(p)} \alpha(m)$ for all $m \in M, p \in P$. We denote by XLie the category of crossed modules of Lie algebras.

There are several equivalent descriptions of the category XLie. We mention here the equivalences with simplicial Lie algebras whose Moore complexes are of length 1 , with internal categories in the category of Lie algebras and with cat ${ }^{1}$-Lie algebras. The nature of these equivalences is reminiscent of the interplays between similar objects in groups [20]. The equivalence between Lie crossed modules and cat $^{1}$-Lie algebras will be used in what follows and we shall give some of its details immediately below.

Recall that a cat ${ }^{1}$-Lie algebra $\left(L_{1}, L_{0}, s, t\right)$ consists of a Lie algebra $L_{1}$ together with a Lie subalgebra $L_{0}$ and structural homomorphisms $s, t: L_{1} \rightarrow L_{0}$ satisfying

$$
\left.s\right|_{L_{0}}=\left.t\right|_{L_{0}}=\operatorname{id}_{L_{0}} \quad \text { and } \quad[\operatorname{Ker} s, \operatorname{Ker} t]=0 .
$$

Given a Lie crossed module $(M, P, \mu)$, the corresponding cat ${ }^{1}$-Lie algebra is $(M \rtimes$ $P, P, s, t)$, where $s(m, p)=p, t(m, p)=\mu(m)+p$ for all $(m, p) \in M \rtimes P$. On the other hand, for a cat ${ }^{1}$-Lie algebra $\left(L_{1}, L_{0}, s, t\right)$ the corresponding Lie crossed module is $\left.t\right|_{\text {Ker } s}:$ Ker $s \rightarrow L_{0}$ with the action of $L_{0}$ on Ker $s$ defined by the Lie bracket in $L_{1}$.

\subsection{Actions and semi-direct product of Lie crossed modules}

In article [22], actor crossed modules of groups are constructed, which provide an analogue of the automorphism group of groups and are used to define actions of crossed modules of groups.

The similar construction for Lie crossed modules is given in [10], and an action of a Lie crossed module $(H, G, \partial)$ on another Lie crossed module $(M, P, \mu)$ is defined to be a morphism $(\alpha, \beta):(H, G, \partial) \rightarrow \operatorname{Act}(M, P, \mu)$ of Lie crossed modules, where

$$
\operatorname{Act}(M, P, \mu)=(\operatorname{Der}(P, M), \operatorname{Der}(M, P, \mu), \Delta)
$$

is the actor crossed module of $(M, P, \mu)$.

Let us recall briefly from [10] the construction of $\operatorname{Act}(M, P, \mu)$. First note that all results in [10] are achieved when the ground ring $K$ is a field, although all constructions work for $K$ a commutative unital ring. Thus, for the Lie crossed module $(M, P, \mu), \operatorname{Der}(P, M)$ denotes the $K$-module of all derivations from $P$ to $M$, i.e., $K$-linear maps $d: P \rightarrow M$ such that $d\left[p, p^{\prime}\right]={ }^{p} d\left(p^{\prime}\right)-p^{\prime} d(p), p, p^{\prime} \in P$. There is a multiplication in $\operatorname{Der}(P, M)$ given by

$$
\left[d_{1}, d_{2}\right]=d_{1} \mu d_{2}-d_{2} \mu d_{1}
$$

turning $\operatorname{Der}(P, M)$ into a Lie algebra. Further, $\operatorname{Der}(M, P, \mu)$ denotes the Lie algebra of derivations of the crossed module $(M, P, \mu)$, i.e., the elements are all pairs $(\phi, \psi)$ with $\phi \in \operatorname{Der}(M), \psi \in \operatorname{Der}(P)$ such that

$$
\psi \mu=\mu \phi \quad \text { and } \quad \phi\left({ }^{p} m\right)={ }^{p} \phi(m)+{ }^{\psi(p)} m
$$

for all $m \in M, p \in P$. The Lie structure of $\operatorname{Der}(M, P, \mu)$ is given simply by

$$
\begin{aligned}
\left(\phi_{1}, \psi_{1}\right)+\left(\phi_{2}, \psi_{2}\right) & =\left(\phi_{1}+\phi_{2}, \psi_{1}+\psi_{2}\right), \\
\lambda(\phi, \psi) & =(\lambda \phi, \lambda \psi), \\
{\left[\left(\phi_{1}, \psi_{1}\right),\left(\phi_{2}, \psi_{2}\right)\right] } & =\left(\left[\phi_{1}, \phi_{2}\right],\left[\psi_{1}, \psi_{2}\right]\right),
\end{aligned}
$$

for all $\left(\phi_{1}, \psi_{1}\right),\left(\phi_{2}, \psi_{2}\right),(\phi, \psi) \in \operatorname{Der}(M, P, \mu), \lambda \in K$. Then direct calculations show 
that the Lie homomorphism

$$
\Delta: \operatorname{Der}(P, M) \rightarrow \operatorname{Der}(M, P, \mu), \quad d \mapsto(d \mu, \mu d),
$$

together with the Lie action of $\operatorname{Der}(M, P, \mu)$ on $\operatorname{Der}(P, M)$ given by

$$
{ }^{(\phi, \psi)} d=\phi d-d \psi
$$

is a Lie crossed module called the actor of $(M, P, \mu)$, denoted by $\operatorname{Act}(M, P, \mu)$.

Example 2.1. There is an action of a Lie crossed module $(M, P, \mu)$ on itself given by the morphism $\left(\alpha_{\mu}, \beta_{\mu}\right):(M, P, \mu) \rightarrow \operatorname{Act}(M, P, \mu)$, where $\alpha_{\mu}: M \rightarrow \operatorname{Der}(P, M)$ is defined by $\alpha_{\mu}(m)(p)=-{ }^{p} m$, and $\beta_{\mu}: P \rightarrow \operatorname{Der}(M, P, \mu)$ is defined by $\beta_{\mu}(p)=$ $\left(\phi_{p}, \psi_{p}\right)$ with $\phi_{p}(m)={ }^{p} m$ and $\psi_{p}\left(p^{\prime}\right)=\left[p, p^{\prime}\right]$ for all $m \in M, p, p^{\prime} \in P$. Moreover, the kernel of $\left(\alpha_{\mu}, \beta_{\mu}\right)$ is the center of the Lie crossed module $(M, P, \mu)$ (see more details in $[\mathbf{1 0}])$.

There is an equivalent description of actions of Lie crossed modules.

Proposition 2.2. Let $(H, G, \partial)$ and $(M, P, \mu)$ be Lie crossed modules. An action of $(H, G, \partial)$ on $(M, P, \mu)$ is determined by the following conditions:

(i) There are actions of the Lie algebra $G$ (and so $H$ ) on the Lie algebras $M$ and $P ; \mu$ is a G-equivariant homomorphism, that is, $\mu\left({ }^{g} m\right)={ }^{g} \mu(m)$; and $G$, $P$ act compatibly on $M$, that is,

$$
{ }^{\left({ }^{p}\right)} m={ }^{g}\left({ }^{p} m\right)-{ }^{p}\left({ }^{g} m\right)
$$

for all $g \in G, p \in P$, and $m \in M$.

(ii) There is a K-bilinear map $f: H \times P \rightarrow M$ such that, for $h, h^{\prime} \in H, p, p^{\prime} \in P$, $m \in M, g \in G$,

$$
\begin{aligned}
f(h, \mu(m)) & ={ }^{h} m, \\
\mu f(h, p) & ={ }^{h} p, \\
{ }^{g} f(h, p) & =f\left({ }^{g} h, p\right)+f\left(h,{ }^{g} p\right), \\
f\left(\left[h, h^{\prime}\right], p\right) & ={ }^{h} f\left(h^{\prime}, p\right)-{ }^{h^{\prime}} f(h, p), \\
f\left(h,\left[p, p^{\prime}\right]\right) & ={ }^{p} f\left(h, p^{\prime}\right)-{ }^{p^{\prime}} f(h, p) .
\end{aligned}
$$

Proof. Let $(H, G, \partial)$ act on $(M, P, \mu)$ by the following morphism of Lie crossed modules:

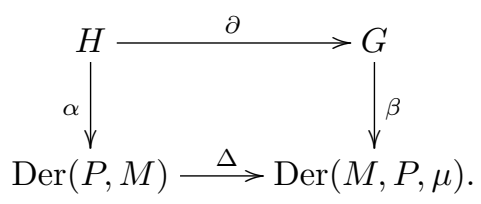

Clearly the homomorphism $\beta$ defines Lie actions of $G$ on $M$ and $P, \mu$ is $G$-equivariant because of the first equality in (2), and (3) is a consequence of the second equality in (2). Define $f: H \times P \rightarrow M$ by $f(h, p)=\alpha(h)(p)$. Then the commutativity of the diagram (9) amounts precisely to the equalities (4) and (5); the equality (6) is equivalent to $\alpha\left({ }^{g} h\right)={ }^{\beta(g)} \alpha(h)$; whilst (7) and (8) are equivalent to the facts that $\alpha$ is a Lie homomorphism and $\alpha(g)$, for any $g \in G$, is a derivation, respectively. The inverse statement is obvious. 
Let $(H, G, \partial)$ be a Lie crossed module acting on a Lie crossed module $(M, P, \mu)$. By Proposition 2.2 there are Lie algebra actions of $H$ on $M$ and of $G$ on $P$. With these actions we can form the semi-direct products of Lie algebras $M \rtimes H$ and $P \rtimes G$.

Lemma 2.3. There is an action of the Lie algebra $P \rtimes G$ on the Lie algebra $M \rtimes H$ such that, with this action, the Lie homomorphism

$$
(\mu, \partial): M \rtimes H \rightarrow P \rtimes G,(\mu, \partial)(m, h)=(\mu(m), \partial(h)),(m, h) \in M \rtimes H,
$$

is a Lie crossed module.

Proof. Using the same notations as in Proposition 2.2, the action of $P \rtimes G$ on $M \rtimes H$ is defined by

$$
{ }^{(p, g)}(m, h)=\left({ }^{p} m+{ }^{g} m-f(h, p),{ }^{g} h\right)
$$

for all $p \in P, g \in G, m \in M$, and $h \in H$. The remaining details are routine calculations.

The Lie crossed module $(M \rtimes H, P \rtimes G,(\mu, \partial))$ is called the semi-direct product of Lie crossed modules $(M, P, \mu)$ and $(H, G, \partial)$. Note that the semi-direct product determines a split extension of $(H, G, \partial)$ by $(M, P, \mu)$

$$
(0,0,0) \longrightarrow(M, P, \mu) \longrightarrow(M \rtimes H, P \rtimes G,(\mu, \partial)) \rightleftarrows(H, G, \partial) \longrightarrow(0,0,0)
$$

with the splitting morphism being the pair of inclusions $(H \hookrightarrow M \rtimes H, G \hookrightarrow P \rtimes G)$. And conversely, any split extension of $(H, G, \partial)$ by $(M, P, \mu)$ is isomorphic to their semi-direct product, where the action of $(H, G, \partial)$ on $(M, P, \mu)$ is induced by the splitting morphism (see $[\mathbf{9}]$ ).

Remark 2.4. A general theory of split short exact sequences, semi-direct products, and actions exists in the context of semi-abelian categories (see [4]) and of course those notions agree with the ad-hoc definitions given here.

\subsection{Crossed modules of algebras and cat $^{1}$-algebras} $[14])$.

We recall the basic notions about crossed modules of algebras from [12] (also cf.

We say that an algebra $A$ acts on another algebra $R$ if, as a $K$-module, $R$ has an $A$-bimodule structure $A \times R \rightarrow R,(a, r) \mapsto a \cdot r, R \times A \rightarrow R,(r, a) \mapsto r \cdot a$, and the following conditions are verified:

$$
a \cdot\left(r r^{\prime}\right)=(a \cdot r) r^{\prime}, \quad(r \cdot a) r^{\prime}=r\left(a \cdot r^{\prime}\right), \quad\left(r r^{\prime}\right) \cdot a=r\left(r^{\prime} \cdot a\right),
$$

for all $a \in A, r, r^{\prime} \in R$. For example, if $A$ is a subalgebra of some algebra $B$ and $R$ is an ideal in $B$, then multiplication in $B$ yields an action of $A$ on $R$.

Given an action of $A$ on $R$, one can form the semi-direct product algebra, $R \rtimes A$, with the underlying $K$-module $R \oplus A$ endowed with the multiplication given by

$$
(r, a)\left(r^{\prime}, a^{\prime}\right)=\left(r r^{\prime}+a \cdot r^{\prime}+r \cdot a^{\prime}, a a^{\prime}\right)
$$

for all $(r, a),\left(r^{\prime}, a^{\prime}\right) \in R \rtimes A$. 
A crossed module of algebras $(R, A, \rho)$ is an algebra homomorphism $\rho: R \rightarrow A$, together with an action of $A$ on $R$, such that the following conditions hold:

$$
\begin{aligned}
& \rho(a \cdot r)=a \rho(r), \quad \rho(r \cdot a)=\rho(r) a, \\
& \rho(r) \cdot r^{\prime}=r r^{\prime}=r \cdot \rho\left(r^{\prime}\right),
\end{aligned}
$$

for all $a \in A, r, r^{\prime} \in R$.

The concept of a crossed module of algebras generalizes simultaneously the concepts of an ideal as well as a bimodule. In fact, a common instance of a crossed module of algebras is that of an algebra $A$ possessing an ideal $I$; the inclusion homomorphism $I \hookrightarrow A$ is a crossed module with $A$ acting on $I$ by the multiplication in $A$.

Another common example is that of an $A$-bimodule $M$ with trivial multiplication; then the zero homomorphism $0: M \rightarrow A, m \mapsto 0$, is a crossed module.

Any epimorphism of algebras $R \rightarrow A$ with the kernel in the two-sided annihilator of $R$ is a crossed module, with $a \in A$ acting on $r \in R$ by $a \cdot r=\tilde{r} r$ and $r \cdot a=r \tilde{r}$, where $\tilde{r}$ is any element in the preimage of $a$.

One more example is that of a DG-algebra concentrated in degrees 0 and $1, A=$ $\left\{A_{1} \stackrel{d}{\longrightarrow} A_{0}\right\}$, with $A_{0}$ acting on $A_{1}$ by multiplication in $A$.

A morphism $(\alpha, \beta):(R, A, \rho) \rightarrow\left(R^{\prime}, A^{\prime}, \rho^{\prime}\right)$ of crossed modules is a pair of homomorphisms $\left(\alpha: R \rightarrow R^{\prime}, \beta: A \rightarrow A^{\prime}\right)$ such that $\rho^{\prime} \alpha=\beta \rho, \alpha(a \cdot r)=\beta(a) \cdot \alpha(r)$, and $\alpha(r \cdot a)=\alpha(r) \cdot \beta(a)$ for $a \in A, r \in R$. Let us denote the category of crossed modules of algebras by XAlg. Moreover, denote by $\mathbf{X A l g}{ }^{1}$ the subcategory of XAlg of crossed modules of unital algebras, whose objects are crossed modules $(R, A, \rho)$ with $A$ a unital algebra and whose morphisms are crossed module morphisms $(\alpha, \beta)$ with $\beta$ a homomorphism of unital algebras.

A cat ${ }^{1}$-algebra $\left(A_{1}, A_{0}, \sigma, \tau\right)$ consists of an algebra $A_{1}$ together with a subalgebra $A_{0}$ and structural homomorphisms $\sigma, \tau: A_{1} \rightarrow A_{0}$ satisfying

$$
\left.\sigma\right|_{A_{0}}=\left.\tau\right|_{A_{0}}=\operatorname{id}_{A_{0}} \quad \text { and } \quad \operatorname{Ker} \sigma \operatorname{Ker} \tau=0=\operatorname{Ker} \tau \operatorname{Ker} \sigma .
$$

A morphism of cat $^{1}$-algebras $\gamma:\left(A_{1}, A_{0}, \sigma, \tau\right) \rightarrow\left(A_{1}^{\prime}, A_{0}^{\prime}, \sigma^{\prime}, \tau^{\prime}\right)$ is an algebra homomorphism $\gamma: A_{1} \rightarrow A_{1}^{\prime}$ such that $\gamma\left(A_{0}\right) \subseteq A_{0}^{\prime}$ and $\sigma^{\prime} \gamma=\left.\gamma\right|_{A_{0}} \sigma, \tau^{\prime} \gamma=\left.\gamma\right|_{A_{0}} \tau$.

Just as in the case of Lie algebras, a crossed module of algebras is equivalent to a cat $^{1}$-algebra. More precisely, given a crossed module of algebras $(R, A, \rho)$, the corresponding cat ${ }^{1}$-algebra is $(R \rtimes A, A, \sigma, \tau)$, where $\sigma(r, a)=a, \tau(r, a)=\rho(r)+a$ for all $(r, a) \in R \rtimes A$. On the other hand, for a cat ${ }^{1}$-algebra $\left(A_{1}, A_{0}, \sigma, \tau\right)$ the corresponding crossed module is $\left.\tau\right|_{\operatorname{Ker} \sigma}$ : $\operatorname{Ker} \sigma \rightarrow A_{0}$ with the action of $A_{0}$ on $\operatorname{Ker} \sigma$ defined by the multiplication in $A_{1}$.

\section{Enveloping crossed module and adjunction between XLie and XAlg}

Any algebra $A$ becomes a Lie algebra with the Lie bracket $[a, b]=a b-b a, a, b \in A$. Let Lie: Alg $\rightarrow$ Lie denote the functor sending an algebra $A$ to its Lie algebra $\operatorname{Lie}(A)$. Let $\mathrm{U}: \mathbf{L i e} \rightarrow \mathbf{A l g}$ denote its left adjoint functor, sending a Lie algebra $L$ to its universal enveloping algebra $\mathrm{U}(L)$. In this section we extend the functors Lie and $\mathrm{U}$ to the categories XAlg and XLie in such a way that those extensions are still adjoint to one another. 


\subsection{Liezation of crossed modules of algebras}

As noted in [12], we can associate to a crossed module of algebras $(R, A, \rho)$ the Lie crossed module $(\operatorname{Lie}(R), \operatorname{Lie}(A), \operatorname{Lie}(\rho))$ with the action of $\operatorname{Lie}(A)$ on $\operatorname{Lie}(R)$ given by ${ }^{a} r=a \cdot r-r \cdot a$ for all $a \in A, r \in R$. It is easy to see that this is indeed a Lie action, and $(\operatorname{Lie}(R), \operatorname{Lie}(A), \operatorname{Lie}(\rho))$ is a Lie crossed module. Moreover, this assignment defines a functor XLie: XAlg $\rightarrow$ XLie which is a natural generalization of the functor Lie: $\mathbf{A l g} \rightarrow$ Lie in the following sense. There are full embeddings

$$
\mathbb{I}_{0}, \mathbb{I}_{1}: \text { Alg } \longrightarrow \text { XAlg } \quad \text { (resp. } \mathbb{I}_{0}^{\prime}, \mathbb{I}_{1}^{\prime}: \text { Lie } \longrightarrow \text { XLie) }
$$

defined, for an algebra $A$ (resp. Lie algebra $P)$, by $\mathbb{I}_{0}(A)=(0, A, 0), \mathbb{I}_{1}(A)=\left(A, A, \operatorname{id}_{A}\right)$ $\left(\right.$ resp. $\left.\mathbb{I}_{0}^{\prime}(P)=(0, P, 0), \mathbb{I}_{1}^{\prime}(P)=\left(P, P, \mathrm{id}_{P}\right)\right)$, and it is immediate to see that we have the following commutative diagram

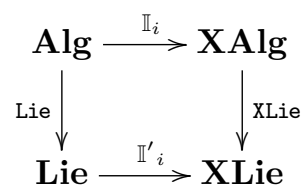

for $i=0,1$.

\subsection{Universal enveloping crossed module}

Now we construct a left adjoint functor to the functor XLie, which generalizes the universal enveloping algebra functor U: $\mathbf{L i e} \rightarrow \mathbf{A l g}$ to crossed modules.

Given a Lie crossed module $\mu: M \rightarrow P$, consider its corresponding cat $^{1}$-Lie algebra $M \rtimes P \underset{t}{\stackrel{s}{\rightleftarrows}} P$. Then, applying the universal enveloping algebra functor $\mathrm{U}$, we obtain a diagram of algebras

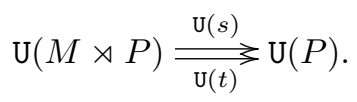

It is clear that $\left.\mathrm{U}(s)\right|_{U(P)}=\left.\mathrm{U}(t)\right|_{U(P)}=\mathrm{id}_{\mathrm{U}(P)}$, but the kernel condition in the definition of a cat ${ }^{1}$-algebra is not fulfilled in general. Therefore, we consider a new diagram

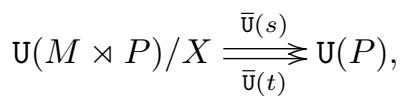

where $X=\operatorname{Ker} \mathrm{U}(s) \operatorname{Ker} \mathrm{U}(t)+\operatorname{Ker} \mathrm{U}(t) \operatorname{Ker} \mathrm{U}(s), \overline{\mathrm{U}}(s)$, and $\overline{\mathrm{U}}(t)$ are induced by $\mathrm{U}(s)$ and $\mathrm{U}(t)$, respectively. This obviously is a cat ${ }^{1}$-algebra.

Define $\mathrm{XU}(M, P, \mu)$ as the crossed module of algebras corresponding to the cat ${ }^{1}$ algebra (11), that is,

$$
\mathrm{XU}(M, P, \mu)=\left(\operatorname{Ker} \overline{\mathrm{U}}(s), \mathrm{U}(P),\left.\overline{\mathrm{U}}(t)\right|_{\operatorname{Ker} \overline{\mathrm{U}}(s)}\right) .
$$

Note that, in fact, $\mathrm{XU}(M, P, \mu)$ is an object of $\mathbf{X} \mathbf{A} \mathbf{l g}^{1}$.

Definition 3.1. Given a Lie crossed module $(M, P, \mu)$, the crossed module of algebras $\mathrm{XU}(M, P, \mu)$ is called the universal enveloping crossed module of $(M, P, \mu)$.

It is easy to see that the universal enveloping crossed module construction provides a functor XU: $\mathbf{X L i e} \rightarrow \mathbf{X A l g}$, which is a natural generalization of the functor $\mathrm{U}$ in 
the sense that the diagram

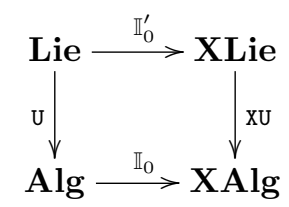

is commutative. Moreover, one has the following

Proposition 3.2. There is a natural isomorphism of functors

$$
\mathbb{I}_{1} \circ \mathrm{U} \approx \mathrm{XU} \circ \mathbb{I}_{1}^{\prime}
$$

Proof. Given a Lie algebra $P$, we have that $\mathbb{I}_{1} \circ \mathrm{U}(P)=\left(\mathrm{U}(P), \mathrm{U}(P), \mathrm{id}_{\mathrm{U}(P)}\right)$. Thus, we need to show that the crossed module of algebras $\mathrm{XU}\left(P, P, \mathrm{id}_{P}\right) \quad\left(=\mathrm{XU} \circ \mathbb{I}_{1}^{\prime}(P)\right)$ is isomorphic to $\left(\mathrm{U}(P), \mathrm{U}(P), \mathrm{id}_{\mathrm{U}(P)}\right)$. We follow the definition of $\mathrm{XU}$ and consider the cat ${ }^{1}$-Lie algebra $(P \rtimes P, P, s, t)$ with $s\left(p, p^{\prime}\right)=p^{\prime}, t\left(p, p^{\prime}\right)=p+p^{\prime}, p, p^{\prime} \in P$, corresponding to the Lie crossed module $\left(P, P, \mathrm{id}_{P}\right)$. There is a Lie homomorphism $\epsilon: P \rightarrow P \rtimes P, p \mapsto(p, 0)$, satisfying $s \epsilon=0$ and $t \epsilon=\mathrm{id}_{P}$. Next, we need to consider the cat ${ }^{1}$-algebra $(\mathrm{U}(P \rtimes P) / X, \mathrm{U}(P), \overline{\mathrm{U}}(s), \overline{\mathrm{U}}(t))$, where $X=\operatorname{Ker} \mathrm{U}(s) \operatorname{Ker} \mathrm{U}(t)+$ $\operatorname{Ker} \mathrm{U}(t) \operatorname{Ker} \mathrm{U}(s)$.

Let $\pi: \mathrm{U}(P \rtimes P) \rightarrow \mathrm{U}(P \rtimes P) / X$ denote the canonical epimorphism. Then we have the equalities $\overline{\mathrm{U}}(s) \pi \mathrm{U}(\epsilon)=\mathrm{U}(s) \mathrm{U}(\epsilon)=0$ and $\overline{\mathrm{U}}(t) \pi \mathrm{U}(\epsilon)=\mathrm{U}(t) \mathrm{U}(\epsilon)=\mathrm{id}_{P}$.

It follows that the homomorphism $\pi \mathrm{U}(\epsilon)$ takes values in $\operatorname{Ker} \overline{\mathrm{U}}(s)$ and it is a right inverse for the homomorphism $\left.\overline{\mathrm{U}}(t)\right|_{\operatorname{Ker} \overline{\mathrm{U}}(s)}: \operatorname{Ker} \overline{\mathrm{U}}(s) \rightarrow \mathrm{U}(P)$.

Now we show that $\left.\pi \mathrm{U}(\epsilon) \overline{\mathrm{U}}(t)\right|_{\operatorname{Ker} \overline{\mathrm{U}}(s)}=\operatorname{id}_{\operatorname{Ker} \overline{\mathrm{U}}(s)}$. Indeed, we have that $\operatorname{Ker} \overline{\mathrm{U}}(s)=$ $\operatorname{Ker} \mathrm{U}(s) / X$ and, as a $K$-module, $\operatorname{Ker} \mathrm{U}(s)$ is generated by all elements of the form

$$
\left(p_{1}, p_{1}^{\prime}\right) \otimes \cdots \otimes\left(p_{i-1}, p_{i-1}^{\prime}\right) \otimes\left(p_{i}, 0\right) \otimes\left(p_{i+1}, p_{i+1}^{\prime}\right) \otimes \cdots \otimes\left(p_{n}, p_{n}^{\prime}\right)
$$

with $\left(p_{i}, p_{i}^{\prime}\right) \in P \rtimes P, 1 \leqslant i \leqslant n$. By definitions of $\mathrm{U}(t)$ and $\mathrm{U}(\epsilon)$, the value of $\mathrm{U}(\epsilon) \mathrm{U}(t)$ on $(12)$ is

$$
\left(p_{1}+p_{1}^{\prime}, 0\right) \otimes \cdots \otimes\left(p_{i-1}+p_{i-1}^{\prime}, 0\right) \otimes\left(p_{i}, 0\right) \otimes\left(p_{i+1}+p_{i+1}^{\prime}, 0\right) \otimes \cdots \otimes\left(p_{n}+p_{n}^{\prime}, 0\right) .
$$

Then we easily derive the following equalities in $\operatorname{Ker} \mathrm{U}(s) / X$ :

$$
\begin{aligned}
& \left(p_{1}+p_{1}^{\prime}, 0\right) \otimes \cdots \otimes\left(p_{i-1}+p_{i-1}^{\prime}, 0\right) \otimes\left(p_{i}, 0\right) \otimes\left(p_{i+1}+p_{i+1}^{\prime}, 0\right) \otimes \cdots \otimes\left(p_{n}+p_{n}^{\prime}, 0\right) \\
& =\left(p_{1}, p_{1}^{\prime}\right) \otimes \cdots \otimes\left(p_{i-1}+p_{i-1}^{\prime}, 0\right) \otimes\left(p_{i}, 0\right) \otimes\left(p_{i+1}+p_{i+1}^{\prime}, 0\right) \otimes \cdots \otimes\left(p_{n}+p_{n}^{\prime}, 0\right) \\
& =\cdots \\
& =\left(p_{1}, p_{1}^{\prime}\right) \otimes \cdots \otimes\left(p_{i-1}, p_{i-1}^{\prime}\right) \otimes\left(p_{i}, 0\right) \otimes\left(p_{i+1}, p_{i+1}^{\prime}\right) \otimes \cdots \otimes\left(p_{n}, p_{n}^{\prime}\right) .
\end{aligned}
$$

Thus, the elements (12) and (13) are equal in $\operatorname{Ker} \mathrm{U}(s) / X$ and it follows that

$$
\left.\pi \mathrm{U}(\epsilon) \overline{\mathrm{U}}(t)\right|_{\operatorname{Ker} \overline{\mathrm{U}}(s)}=\mathrm{id}_{\operatorname{Ker} \overline{\mathrm{U}}(s)} .
$$

Now it is easy to see that the pair $\left(\left.\overline{\mathrm{U}}(t)\right|_{\operatorname{Ker} \overline{\mathrm{U}}(s)}, \mathrm{id}_{\mathrm{U}(P)}\right)$ is an isomorphism between the crossed modules of algebras $\left(\operatorname{Ker} \overline{\mathrm{U}}(s), \mathrm{U}(P),\left.\overline{\mathrm{U}}(t)\right|_{\operatorname{Ker} \mathrm{U}(s)}\right)$ and $\left(\mathrm{U}(P), \mathrm{U}(P), \operatorname{id}_{\mathrm{U}(P)}\right)$, which provides the required isomorphism between the functors $\mathbb{I}_{1} \circ \mathrm{U}$ and $\mathrm{XU} \circ \mathbb{I}_{1}^{\prime}$.

Remark 3.3. In the article [15], because of the integration problem of Lie 2-algebras into 2-groups, an enveloping functor $\mathbb{U}$ is defined by applying the standard functor $U$ 
term by term on a Lie crossed module $(M, P, \mu)$, i.e., $\mathbb{U}(M, P, \mu)=(\mathrm{U}(M), \mathrm{U}(P), \mathrm{U}(\mu))$. Also, it is noted that this does not give a crossed module of associative algebras, but a crossed module of Hopf algebras, presented in [15, Definition 1]. Of course, our definition of the universal enveloping functor XU differs from the definition of the enveloping functor $\mathbb{U}$ in $[\mathbf{1 5}]$.

\subsection{Adjunction between XLie and XAlg}

The following result is a natural generalization of the well-known classical adjunction between the categories Lie and Alg.

Theorem 3.4. The functor $\mathrm{XU}$ is left adjoint to the Liezation functor XLie.

Proof. We have to construct a natural bijection

$$
\operatorname{Hom}_{\mathbf{X L i e}}((M, P, \mu), \mathrm{XLie}(R, A, \rho)) \approx \operatorname{Hom}_{\mathbf{X A l g}}(\mathrm{XU}(M, P, \mu),(R, A, \rho)),
$$

for any $(M, P, \mu) \in$ XLie and $(R, A, \rho) \in$ XAlg.

Given a morphism $(\alpha, \beta) \in \operatorname{Hom}_{\mathbf{X L i e}}((M, P, \mu), \operatorname{XLie}(R, A, \rho))$, consider the corresponding morphism of cat $^{1}$-Lie algebras

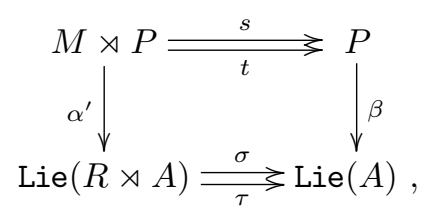

where $\alpha^{\prime}(m, p)=(\alpha(m), \beta(p))$ for all $(m, p) \in M \rtimes P$. Since the functor $\mathrm{U}$ is left adjoint to the functor Lie, we easily deduce that there is an induced commutative diagram of algebras

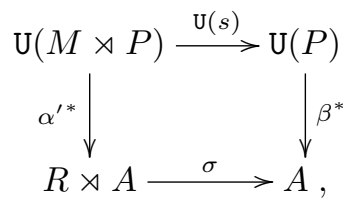

and a similar diagram holds with $\mathrm{U}(s)$ replaced by $\mathrm{U}(t)$ and $\sigma$ replaced by $\tau$. Since $\operatorname{Ker} \sigma \operatorname{Ker} \tau=\operatorname{Ker} \tau \operatorname{Ker} \sigma=0$, we have a uniquely defined morphism of cat ${ }^{1}$-algebras

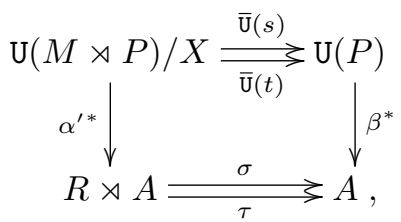

which corresponds to a uniquely defined morphism from $\operatorname{Hom} \mathbf{X A l g}(\mathrm{XU}(M, P, \mu)$, $(R, A, \rho))$. The inverse assignment is obvious.

Let us remark that the adjunction XU $\dashv$ XLie actually follows from a simple composition of right adjoint functors which determines the left adjoint functor XU completely. 
More concretely, let us consider the following diagram in which the respective squares of left and right adjoint functors are commutative.

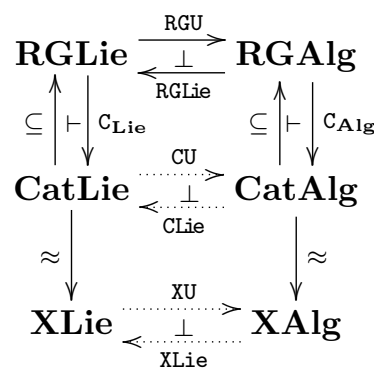

Here RGLie (resp. RGAlg) is the category of reflexive graphs in Lie (resp. Alg). The functor RGU (resp. RGLie) is the pointwise extension of U (resp. Lie). CatLie (resp. CatAlg) is the category of internal categories in Lie (resp. Alg), which is equivalent to the category of cat $^{1}$-Lie algebras (resp. cat ${ }^{1}$-algebras) and so to the category XLie (resp. XAlg), and which is reflective in RGLie (resp. RGAlg) with the reflector $\mathrm{C}_{\text {Lie }}$ (resp. $\mathrm{C}_{\mathrm{Alg}}$ ).

The functor CLie is the restriction of RGLie to internal categories. It is right adjoint as a composite of right adjoint functors. This agrees with the description of XLie in Subsection 3.1, because right adjoints commute with taking kernels and hence with the equivalence between internal categories and crossed modules. By comparing the commutator condition in (1) with the corresponding equation in (10), it follows immediately that RGLie of an internal category in Alg is indeed an internal category in Lie.

Since the reflector $\mathbf{C}_{\mathbf{L i e}}$ is a split epimorphism, the (unique) left adjoint CU of CLie must be equal to composition $\mathrm{C}_{\mathrm{Alg}} \circ \mathrm{RGU} \circ \subseteq$, which is precisely the description of $\mathrm{XU}$ given in Subsection 3.2.

\section{Modules over crossed modules}

In this section we describe left modules over crossed modules of Lie and associative algebras and prove an equivalence of categories of modules over a Lie crossed module and its universal enveloping crossed module of associative algebras.

\subsection{Modules over Lie crossed modules}

Recall that Beck $[\mathbf{3}]$ introduced a convenient notion of coefficient module to be used in (co)homology theories. The notion makes sense in a broad context, and recovers the usual notions of modules in familiar settings: for groups, commutative algebras, and Lie algebras, these are left modules; for associative algebras, the appropriate notion is that of bimodule.

By definition, given an object $C$ of a category $\mathcal{C}$, a Beck module over $C$ is an abelian group object in the slice category $\mathcal{C} / C$. If $\mathcal{C}$ is a category of interest in the sense of Orzech [23], then Beck modules are equivalent to split extensions with abelian kernel [23, Theorem 2.7]. This is the case for all aforementioned familiar categories. Moreover, the description of crossed modules in groups as cat ${ }^{1}$-groups makes their category into a category of interest (see e.g. [24]). But the same assertion fails for Lie 
crossed modules. Concretely, the category XLie satisfies all the axioms of a category of interest except one [23, Axiom (6)], which is replaced by a new axiom (see details in $[\mathbf{7}]$ for precrossed modules of Lie algebras). However, since the proof of $[\mathbf{2 3}$, Theorem $2.7]$ does not use the condition in $[\mathbf{2 3}$, Axiom (6)], we can apply this general result to the category XLie and identify a module over a Lie crossed module $(H, G, \mu)$ with a split extension in XLie

$$
(0,0,0) \longrightarrow(M, P, \mu) \longrightarrow\left(H^{\prime}, G^{\prime}, \partial^{\prime}\right) \rightleftarrows(H, G, \partial) \longrightarrow(0,0,0)
$$

where the kernel $(M, P, \mu)$ is an abelian crossed module of Lie algebras, that is, $M, P$ abelian Lie algebras, and trivial action of $P$ on $M\left({ }^{p} m=0\right.$, for all $\left.p \in P, m \in M\right)$. It follows from the discussion at the end of Subsection 2.2 that a left $(H, G, \partial)$ module is equivalent to an abelian Lie crossed module $(M, P, \mu)$ endowed with an $(H, G, \partial)$-action by means of a morphism of Lie crossed modules $(\alpha, \beta):(H, G, \partial) \rightarrow$ $\operatorname{Act}(M, P, \mu)$.

Suppose $\mu: M \rightarrow P$ and $\mu^{\prime}: M^{\prime} \rightarrow P^{\prime}$ are $(H, G, \partial)$-modules with crossed module morphisms $(\varphi, \psi):(H, G, \partial) \rightarrow \operatorname{Act}(M, P, \mu)$ and $\left(\varphi^{\prime}, \psi^{\prime}\right):(H, G, \partial) \rightarrow \operatorname{Act}\left(M^{\prime}, P^{\prime}, \mu^{\prime}\right)$, respectively. Then a morphism from $\mu: M \rightarrow P$ to $\mu^{\prime}: M^{\prime} \rightarrow P^{\prime}$ is a pair of homomorphisms of $K$-modules $\left(f_{M}: M \rightarrow M^{\prime}, f_{P}: P \rightarrow P^{\prime}\right)$ such that

$$
f_{P} \mu=\mu^{\prime} f_{M}, \quad\left(f_{M}, f_{P}\right) \psi(g)=\psi^{\prime}(g)\left(f_{M}, f_{P}\right), \quad f_{M} \varphi(h)=\varphi^{\prime}(h) f_{P},
$$

for all $g \in G$ and $h \in H$. There is an obvious composition of such morphisms and this leads to a definition of the category of $(H, G, \partial)$-modules.

\subsection{Modules over crossed modules of algebras}

It is a classical fact that the categories of (left or right) modules over a Lie algebra and over its universal enveloping algebra are equivalent. As we intend to establish the similar equivalence for categories of modules over a Lie crossed module and over its universal enveloping crossed module, in this subsection we are interested in giving a definition of a left module over a crossed module of algebras. Beck's approach is not useful in this situation. The reason is that a Beck module over an algebra is a bimodule but not a left module over it.

As we know that a left module over an algebra $A$ is a $K$-module $V$ together with an algebra homomorphism from $A$ to the endomorphism algebra $\operatorname{End}_{K}(V)$ of $V$, we were naturally led to search for an adequate construction of an "endomorphism crossed module" such that a left module over a crossed module of algebras would be defined by a crossed module morphism to the endomorphism crossed module. A good reason why our construction is relevant is that the Liezation of the endomorphism crossed module is nothing else but the actor crossed module of Lie algebras (see Lemma 4.6 below). At the same time, it is reasonable to expect a module over a Lie crossed module and its universal enveloping crossed module to be the same.

For this, we take an abelian crossed module of algebras $\delta: V \rightarrow W$; that is, $V$ and $W$ are just $K$-modules considered as algebras with the trivial multiplication and with the trivial action of $W$ on $V$, and $\delta$ is a $K$-homomorphism. Then the $K$-module $\operatorname{Hom}_{K}(W, V)$ is an algebra with the multiplication given, for all $d_{1}, d_{2} \in \operatorname{Hom}_{K}(W, V)$, by

$$
d_{1} * d_{2}=d_{1} \delta d_{2}
$$


Let $\operatorname{End}(V, W, \delta)$ denote the algebra of all pairs $(\phi, \psi)$ with $\phi \in \operatorname{End}_{K}(V)$ and $\psi \in$ $\operatorname{End}_{K}(W)$ such that $\psi \delta=\delta \phi$. It is clear that the map

$$
\theta: \operatorname{Hom}_{K}(W, V) \rightarrow \operatorname{End}(V, W, \delta), \quad d \mapsto(d \delta, \delta d),
$$

is a homomorphism of algebras. Moreover, we have

Lemma 4.1. There is an algebra action of $\operatorname{End}(V, W, \delta)$ on $\operatorname{Hom}_{K}(W, V)$ given by

$$
(\phi, \psi) \cdot d=\phi d \quad \text { and } \quad d \cdot(\phi, \psi)=d \psi
$$

for all $d \in \operatorname{Hom}_{K}(W, V)$ and $(\phi, \psi) \in \operatorname{End}(V, W, \delta)$. Moreover, together with this action, $\left(\operatorname{Hom}_{K}(W, V)\right.$, End $\left.(V, W, \delta), \theta\right)$ is a crossed module of algebras.

Proof. Clearly this is an action of algebras and $\left(\operatorname{Hom}_{K}(W, V), \operatorname{End}(V, W, \delta), \theta\right)$ is a crossed module of algebras because of the following equalities:

$$
\begin{aligned}
\theta((\phi, \psi) \cdot d) & =\theta(\phi d)=(\phi d \delta, \delta \phi d)=(\phi d \delta, \psi \delta d)=(\phi, \psi)(d \delta, \delta d)=(\phi, \psi) \theta(d), \\
\theta(d \cdot(\phi, \psi)) & =\theta(d \psi)=(d \psi \delta, \delta d \psi)=(d \delta \phi, \delta d \psi)=(d \delta, \delta d)(\phi, \psi)=\theta(d)(\phi, \psi), \\
\theta(d) \cdot d^{\prime} & =(d \delta, \delta d) \cdot d^{\prime}=(d \delta) d^{\prime}=d * d^{\prime}=d\left(\delta d^{\prime}\right)=d \cdot\left(d^{\prime} \delta, \delta d^{\prime}\right)=d \cdot \theta\left(d^{\prime}\right)
\end{aligned}
$$

for all $(\phi, \psi) \in \operatorname{End}(V, W, \delta)$ and $d, d^{\prime} \in \operatorname{Hom}_{K}(W, V)$.

Note that $\operatorname{End}(V, W, \delta)$ is a unital algebra and $\left(\operatorname{Hom}_{K}(W, V)\right.$, $\left.\operatorname{End}(V, W, \delta), \theta\right)$ is an object of $\mathbf{X A l g}{ }^{1}$, which is called an endomorphism crossed module.

Definition 4.2. Let $(R, A, \rho)$ be a crossed module of algebras. A left $(R, A, \rho)$-module is an abelian crossed module of algebras $(V, W, \delta)$ together with a morphism $(R, A, \rho) \rightarrow$ $\left(\operatorname{Hom}_{K}(W, V), \operatorname{End}(V, W, \delta), \theta\right)$ of crossed modules of algebras.

Suppose $(V, W, \delta)$ and $\left(V^{\prime}, W^{\prime}, \delta^{\prime}\right)$ are left $(R, A, \rho)$-modules with morphisms of crossed modules of algebras $(\alpha, \beta):(R, A, \rho) \rightarrow\left(\operatorname{Hom}_{K}(W, V), \operatorname{End}(V, W, \partial), \Theta\right)$ and $\left(\alpha^{\prime}, \beta^{\prime}\right):(R, A, \rho) \rightarrow\left(\operatorname{Hom}_{K}\left(W^{\prime}, V^{\prime}\right), \operatorname{End}\left(V^{\prime}, W^{\prime}, \delta^{\prime}\right), \Theta^{\prime}\right)$, respectively.

Then a morphism from $(V, W, \delta)$ to $\left(V^{\prime}, W^{\prime}, \delta^{\prime}\right)$ is a pair of homomorphisms of $K$-modules $\left(f_{V}: V \rightarrow V^{\prime}, f_{W}: W \rightarrow W^{\prime}\right)$ such that

$$
f_{W} \delta=\delta^{\prime} f_{V}, \quad\left(f_{V}, f_{W}\right) \beta(a)=\beta^{\prime}(a)\left(f_{V}, f_{W}\right), \quad f_{V} \alpha(r)=\alpha^{\prime}(r) f_{W},
$$

for all $a \in A$ and $r \in R$. There is an obvious composition of such morphisms and this leads to the definition of the category of $(R, A, \rho)$-modules.

Since crossed modules of algebras and cat $^{1}$-algebras are equivalent, and we have a definition of left modules over a crossed module of algebras, this may also be considered as a left module of the corresponding cat ${ }^{1}$-algebra. However, a direct definition of a left module over a cat ${ }^{1}$-algebra will also be useful and we give it immediately below. First we recall from $[\mathbf{1 6}]$ that a cat ${ }^{1}$-module $\left(V_{1}, V_{0}, i, j\right)$ consists of a $K$ module $V_{1}$, a $K$-submodule $V_{0}$ of $V_{1}$, and structural morphisms $i, j: V_{1} \rightarrow V_{0}$ satisfying $\left.i\right|_{V_{0}}=\left.j\right|_{V_{0}}=\mathrm{id}_{V_{0}}$.

Definition 4.3. A left module over a cat ${ }^{1}$-algebra $\left(A_{1}, A_{0}, \sigma, \tau\right)$ is a cat ${ }^{1}$-module $\left(V_{1}, V_{0}, i, j\right)$ together with a left action of $A_{1}$ on $V_{1}$ and a left action of $A_{0}$ on $V_{0}$ 
such that the structural morphisms commute with the actions, that is, $i\left(a_{1} \cdot v_{1}\right)=$ $\sigma\left(a_{1}\right) \cdot i\left(v_{1}\right), j\left(a_{1} \cdot v_{1}\right)=\tau\left(a_{1}\right) \cdot j\left(v_{1}\right)$ for all $a_{1} \in A_{1}, v_{1} \in V_{1}$, and the condition

$$
\operatorname{Ker} \sigma \cdot \operatorname{Ker} i=0=\operatorname{Ker} \tau \cdot \operatorname{Ker} j
$$

holds, i.e., $a \cdot v=0=a^{\prime} \cdot v^{\prime}$ for all $a \in \operatorname{Ker} \sigma, v \in \operatorname{Ker} i, a^{\prime} \in \operatorname{Ker} \tau, v^{\prime} \in \operatorname{Ker} j$.

Remark 4.4. Note that Forrester-Barker in $[\mathbf{1 6}]$ gave a definition of a left module over a cat ${ }^{1}$-algebra, but omitted the important condition (16), which is essential for proving that this notion is equivalent to a left module over the corresponding crossed module of algebras.

By complete analogy with [1, Proposition 8], one can show that cat ${ }^{1}$-modules are equivalent to 2 -term chain complexes of $K$-modules. This is very similar to the above equivalence between cat ${ }^{1}$-algebras (cat ${ }^{1}$-Lie algebras) and crossed modules of algebras (Lie algebras). A 2-term chain complex of $K$-modules is none other than a $K$-homomorphism $\delta: V \rightarrow W$. This last one can be considered as an abelian crossed module of algebras, and the corresponding cat ${ }^{1}$-module is $(V \oplus W, W, i, j)$, where $i(v, w)=w$ and $j(v, w)=\delta(v)+w$ for all $v \in V, w \in W$.

Theorem 4.5. Let $(R, A, \rho)$ be a crossed module of algebras. An abelian crossed module of algebras $(V, W, \delta)$ is a left module over $(R, A, \rho)$ if and only if the corresponding cat $^{1}$-module $(V \oplus W, W, i, j)$ is a left module over the corresponding cat $^{1}$-algebra $(R \rtimes A, A, \sigma, \tau)$.

Proof. Let $(V, W, \delta)$ be a $(R, A, \rho)$-module. Then there is a morphism $(\alpha, \beta)$ of crossed modules of algebras

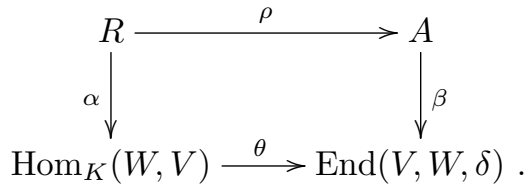

Suppose that $\beta$ has components $\beta_{1}: A \rightarrow \operatorname{End}_{K}(V)$ and $\beta_{2}: A \rightarrow \operatorname{End}_{K}(W)$; that is, $\beta(a)=\left(\beta_{1}(a), \beta_{2}(a)\right)$ for all $a \in A$. It is clear that $A$ acts (to the left) on $V$ and on $W: a \cdot v=\beta_{1}(a)(v)$ and $a \cdot w=\beta_{2}(a)(w)$ for all $a \in A, v \in V, w \in W$. Routine calculations show that the equality

$$
(r, a) \cdot(v, w)=(\alpha(r)(w)+(\rho(r)+a) \cdot v, a \cdot w),
$$

where $(r, a) \in R \rtimes A,(v, w) \in V \oplus W$, defines a left action of the algebra $R \rtimes A$ on the $K$-module $V \oplus W$ and the structural morphisms commute with these actions. In order to check the condition (16), note that $\operatorname{Ker} \sigma(\operatorname{resp} . \operatorname{Ker} \tau, \operatorname{Ker} i, \operatorname{Ker} j)$ consists of all elements of the form $(r, 0)(\operatorname{resp} .(r,-\rho(r)),(v, 0),(v,-\delta(v)))$ for $r \in R, v \in V$, and we have

$$
\begin{aligned}
& (r,-\rho(r)) \cdot(v, 0)=(\alpha(r)(0)+(\rho(r)-\rho(r)) \cdot v, 0)=(0,0), \\
& (r, 0) \cdot(v,-\delta(v))=(-\alpha(r) \delta(v)+\rho(r) \cdot v, 0)=(0,0)
\end{aligned}
$$

since $\alpha(r) \delta(v)=\rho(r) \cdot v$ by commutativity of the diagram (17). 
Conversely, given a left $(R \rtimes A, A, \sigma, \tau)$-module structure on the cat $^{1}$-module $(V \oplus W, W, i, j)$, we define the maps $\alpha$ and $\beta=\left(\beta_{1}, \beta_{2}\right)$ in the diagram (17) by the following equalities:

$$
\begin{aligned}
(\alpha(r)(w), 0) & =(r, 0) \cdot(0, w), \\
\left(\beta_{1}(a)(v), 0\right) & =(0, a) \cdot(v, 0), \\
\beta_{2}(a)(w) & =a \cdot w,
\end{aligned}
$$

for all $r \in R, a \in A, v \in V$, and $w \in W$. Now the remaining details are straightforward calculations and are left to the reader.

\subsection{Equivalence of categories of modules}

We need the following lemma.

Lemma 4.6. Let $\delta: V \rightarrow W$ be a K-homomorphism, viewed as an abelian crossed module of algebras (Lie algebras). Then, using the same notations as in Lemma 4.1, the Lie crossed module $\mathrm{XLie}\left(\operatorname{Hom}_{K}(W, V), \operatorname{End}(V, W, \delta), \theta\right)$ coincides with the actor crossed module $\operatorname{Act}(V, W, \delta)=(\operatorname{Der}(W, V), \operatorname{Der}(V, W, \delta), \Delta)$.

Proof. Since $V$ and $W$ are considered as abelian Lie algebras together with the trivial action of $W$ on $V$, it is clear that $\operatorname{Der}(W, V)=\operatorname{Lie}\left(\operatorname{Hom}_{K}(W, V)\right), \operatorname{Der}(V, W, \delta)=$ Lie $(\operatorname{End}(V, W, \delta))$, and $\Delta=\operatorname{Lie}(\theta)$. Moreover, the Lie action of $\operatorname{Der}(V, W, \delta)$ on $\operatorname{Der}(W, V)$ is induced by the algebra action of $\operatorname{End}(V, W, \delta)$ on $\operatorname{Hom}_{K}(W, V)$.

Theorem 4.7. Let $(M, P, \mu)$ be a Lie crossed module. Then there is an equivalence of categories of left $(M, P, \mu)$-modules and left $\mathrm{XU}(M, P, \mu)$-modules.

Proof. By using Theorem 3.4 and Lemma 4.6, left $(M, P, \mu)$-module structures on a $K$-homomorphism $\delta: V \rightarrow W$ are in bijective correspondence with left $\mathrm{XU}(M, P, \mu)$ module structures on it:

$$
\begin{aligned}
& \operatorname{Hom}_{X \mathbf{L i e}}((M, P, \mu), \operatorname{Act}(V, W, \delta)) \\
& =\operatorname{Hom}_{\mathbf{X L i e}}\left((M, P, \mu), \operatorname{Lie}\left(\operatorname{Hom}_{K}(W, V), \operatorname{End}(V, W, \delta), \theta\right)\right) \\
& \approx \operatorname{Hom}_{\mathbf{X A l g}}\left(\mathrm{XU}(M, P, \mu),\left(\operatorname{Hom}_{K}(W, V), \operatorname{End}(V, W, \delta), \theta\right)\right) .
\end{aligned}
$$

Due to the equations (14) and (15), it is easy to check that this correspondence is functorial.

Finally, let us remark that right modules (over crossed modules of Lie and associative algebras) could be defined similarly and could equally be used everywhere instead of left modules.

\section{Acknowledgments}

The authors would wish to thank the anonymous referee for his help in improving the presentation of this paper. The third author was supported by Xunta de Galicia, grant EM2013/016 (European FEDER support included), and Shota Rustaveli National Science Foundation, grant DI/12/5-103/11. The fourth author was supported by Xunta de Galicia, grant GRC2013-045 (European FEDER support included). The authors were supported by Ministerio de Economa y Competitividad (Spain), grant MTM2013-43687-P (European FEDER support included). 


\section{References}

[1] J.C. Baez and A.S. Crans, Higher-dimensional algebra VI: Lie 2-algebras, Theory Appl. Categ. 12 (2004), 492-538.

[2] H.-J. Baues and E.G Minian, Crossed extensions of algebras and Hochschild cohomology, Homology Homotopy Appl. 4 (2002), 63-82.

[3] J. Beck, Triples, algebras and cohomology, Ph.D. thesis, Columbia University, 1967, Repr. Theory Appl. Categ. 2 (2003), 1-59.

[4] F. Borceux, G. Janelidze and G.M. Kelly, On the representability of actions in a semi-abelian category, Theory Appl. Categ. 14 (2005), 244-286.

[5] R. Brown, Groupoids and crossed objects in algebraic topology, Homology Homotopy Appl. 1 (1999), 1-78.

[6] H. Cartan and S. Eilenberg, Homological algebra, Princeton University Press, Princeton, 1956.

[7] J.M. Casas, T. Datuashvili, M. Ladra and E.O. Uslu, Actions in the category of precrossed modules in Lie algebras, Comm. Algebra 40 (2012), 2962-2982.

[8] J.M. Casas, N. Inassaridze and M. Ladra, Homological aspects of Lie algebra crossed modules, Manuscripta Math. 131 (2010), 385-401.

[9] J.M. Casas and M. Ladra, Perfect crossed modules in Lie algebras, Comm. Algebra 23 (1995), 1625-1644.

[10] J.M. Casas and M. Ladra, The actor of a crossed module in Lie algebras, Comm. Algebra 26 (1998), 2065-2089.

[11] P. Dedecker and A.S.-T. Lue, A nonabelian two-dimensional cohomology for associative algebras, Bull. Amer. Math. Soc. 72 (1966), 1044-1050.

[12] G. Donadze, N. Inassaridze, E. Khmaladze and M. Ladra, Cyclic homologies of crossed modules of algebras, J. Noncommut. Geom. 6 (2012), 749-771.

[13] G. Donadze and M. Ladra, Chevalley-Eilenberg homology of crossed modules of Lie algebras in lower dimensions, Homology Homotopy Appl. 15 (2013), 185-194.

[14] G.J. Ellis, Higher dimensional crossed modules of algebras, J. Pure Appl. Algebra 52 (1988), 277-282.

[15] Y. Frégier and F. Wagemann, On Hopf 2-algebras, Int. Math. Res. Not. IMRN 2011 (2011), 3471-3501.

[16] M. Forrester-Barker, Representations of crossed modules and cat ${ }^{1}$-groups, Ph.D. thesis, University of Bangor, 2003.

[17] D. Guin, Cohomologie des algèbres de Lie croisées et $K$-théorie de Milnor additive, Ann. Inst. Fourier (Grenoble) 45 (1995), 93-118.

[18] N. Inassaridze, E. Khmaladze and M. Ladra, Non-abelian cohomology and extensions of Lie algebras, J. Lie Theory 18 (2008), 413-432.

[19] C. Kassel and J.-L. Loday, Extensions centrales d'algèbres de Lie, Ann. Inst. Fourier (Grenoble) 32 (1982), 119-142.

[20] J.-L. Loday, Spaces with finitely many non-trivial homotopy groups, J. Pure Appl. Algebra 24 (1982), 179-202. 
[21] A.S.-T. Lue, Non-abelian cohomology of associative algebras, Quart. J. Math. Oxford Ser. (2) 19 (1968), 159-180.

[22] K. Norrie, Actions and automorphisms of crossed modules, Bull. Soc. Math. France 118 (1990), 129-146.

[23] G. Orzech, Obstruction theory in algebraic categories I, J. Pure Appl. Algebra 2 (1972), 287-314.

[24] S. Paoli, On the non-balanced property of the category of crossed modules in groups, J. Pure Appl. Algebra 197 (2005), 19-22.

[25] J.H.C. Whitehead, Combinatorial homotopy II, Bull. Amer. Math. Soc. 55 (1949), 453-496.

José Manuel Casas jmcasas@uvigo.es

Department of Applied Mathematics I, University of Vigo, E. E. Forestal, 36005 Pontevedra, Spain

Rafael F. Casado rapha.fdez@gmail.com

Department of Algebra, University of Santiago de Compostela, 15782 Santiago de Compostela, Spain

Emzar Khmaladze e.khmal@gmail.com

A. Razmadze Mathematical Institute, Tbilisi State University, University St. 1, 0186 Tbilisi, Georgia

$\&$

Department of Applied Mathematics I, University of Vigo, E. E. Forestal, 36005 Pontevedra, Spain

Manuel Ladra manuel.ladra@usc.es

Department of Algebra, University of Santiago de Compostela, 15782 Santiago de Compostela, Spain 